\title{
Changes in the Structure and Wear Resistance of the Surface Layer of Copper under Treatment by Nitrogen Ion Beams
}

\author{
Victor P. Sergeev ${ }^{1,2, a)}$, Mark P. Kalashnikov ${ }^{1,2, b)}$, Evgeniya V. Rybalko ${ }^{1, c)}$, \\ Alfred R. Sungatulin ${ }^{1, d)}$, Oleg V. Sergeev ${ }^{1, e)}$, and Stanislav Yu. Zharkov ${ }^{1, \text { f) }}$ \\ ${ }^{1}$ Institute of Strength Physics and Materials Science SB RAS, Tomsk, 634055, Russia \\ ${ }^{2}$ National Research Tomsk Polytechnic University, Tomsk, 634050, Russia

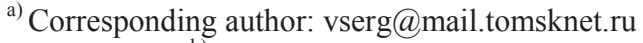 \\ b) kmp1980@mail.ru \\ c) evgeniaribka@yandex.com \\ d) alfred_1972@mail.ru \\ e) tehnovak@ispms.tsc.ru \\ f) zhastas@mail.ru
}

\begin{abstract}
The structural-phase state of the treated sample surface was investigated by TEM. It was shown by the TEM and VIMS method that the improvement of tribological properties of the copper samples can be associated with an increase of relaxation ability due to a significant increase of the nitrogen concentration in it, which is accompanied by the refinement of fcc-Cu main phase grain structure and the formation of nanopores or gas bubbles in the ion-modified surface layer. A high-dose implantation of nitrogen ions and copper samples increases the wear resistance in 1.54.5 times together with a counterbody from the same material in the argon environment. The microhardness of the copper samples also increases.
\end{abstract}

Keywords: wear-resistance, ion implantation, microstructure, phase composition, surface layer, friction couple

\section{INTRODUCTION}

The use of satellite antennas which receive weak electromagnetic signals from outer space under continuous rotation requires electric-friction pairs (EFP) having a high electrical conductivity, a low coefficient of friction and a high wear resistance in the absence of lubrication [1]. Silver and copper alloys are used to produce brushes and collector EFPs. In order to improve the tribological properties of the alloys, molybdenum disulfide, carbon and other elements are added. However, their wear resistance is insufficient.

One method of solving this problem can be ion-beam nanostructuring of the surface layers of the materials, which as shown in $[2,3]$, can significantly improve their tribological properties.

The aim is to study the influence of the surface treatment of the copper samples under high-energy beams by nitrogen ions at high irradiation fluence on their wear resistance and microhardness.

\section{EXPERIMENTAL PROCEDURE}

Nitrogen ions with an energy of $20 \mathrm{keV}$ and fluences from $10^{17}$ to $9 \times 10^{17} \mathrm{~cm}^{-2}$ were implanted into the copper samples using the implanter IGI-3 (Techimlant Ltd.). A sheet copper $5 \times 5 \times 15 \mathrm{~mm}$ in size pre-polished to $\mathrm{Ra}=0.32-$ $0.63 \mu \mathrm{m}$ was used as a material for study. The structural-phase state of the samples was investigated by transmission electron microscopy (TEM) on a microscope JEM-2100 (Jeol Ltd.).

International Conference on Physical Mesomechanics of Multilevel Systems 2014

AIP Conf. Proc. 1623, 559-562 (2014); doi: 10.1063/1.4899006

(C) 2014 AIP Publishing LLC 978-0-7354-1260-6/\$30.00 


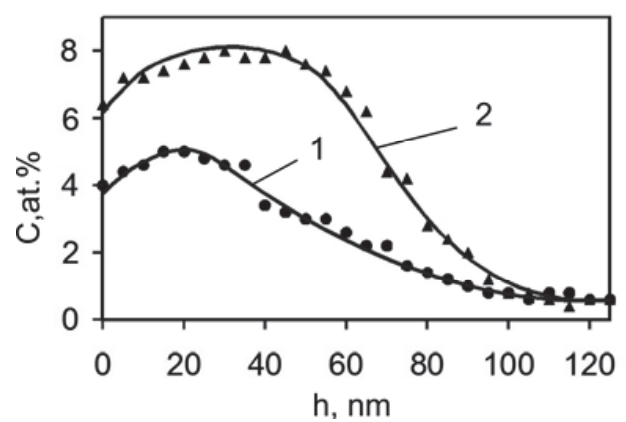

(a)

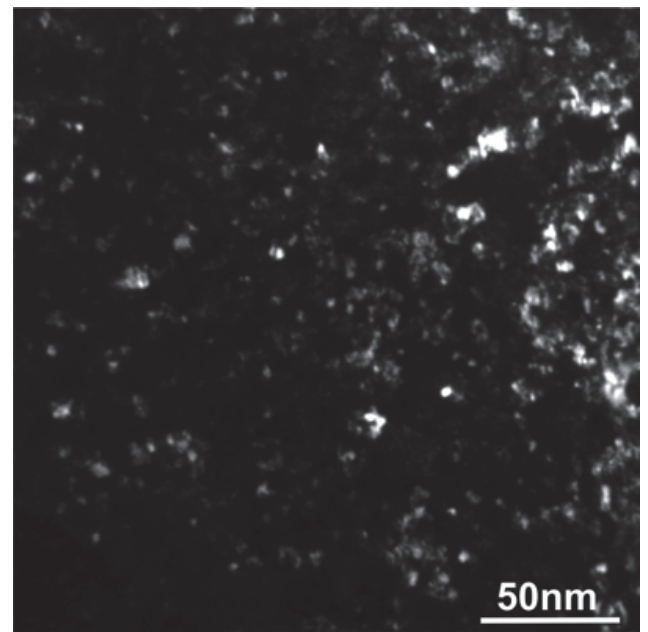

(b)

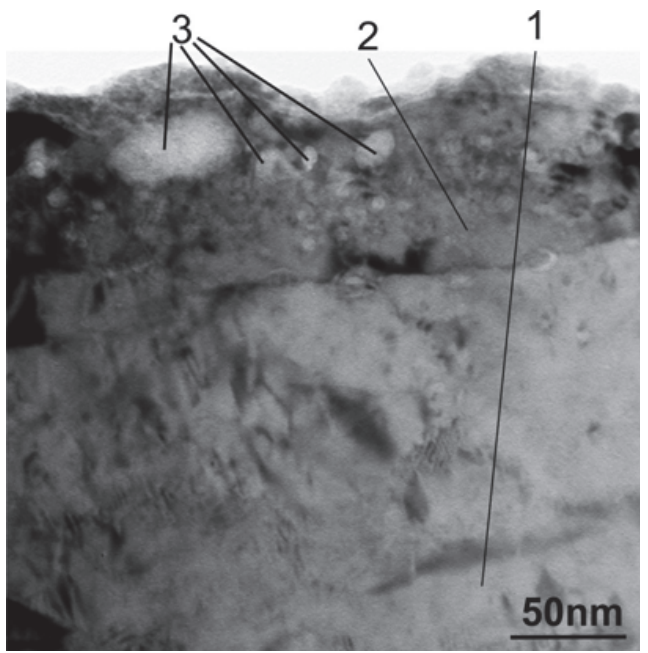

(c)

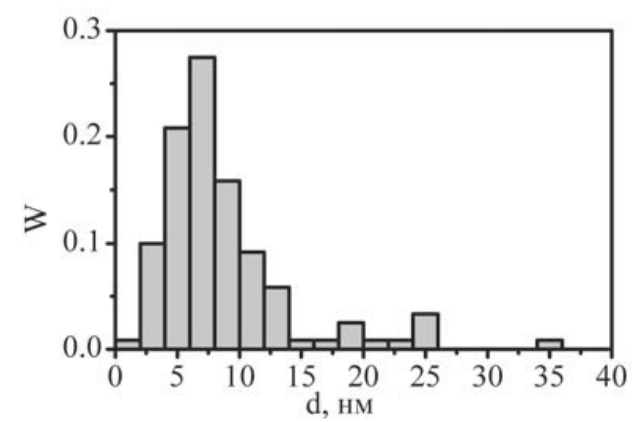

(d)
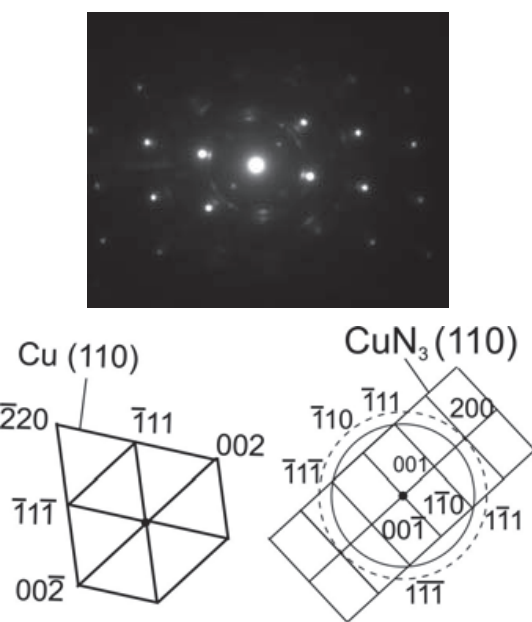

(e)

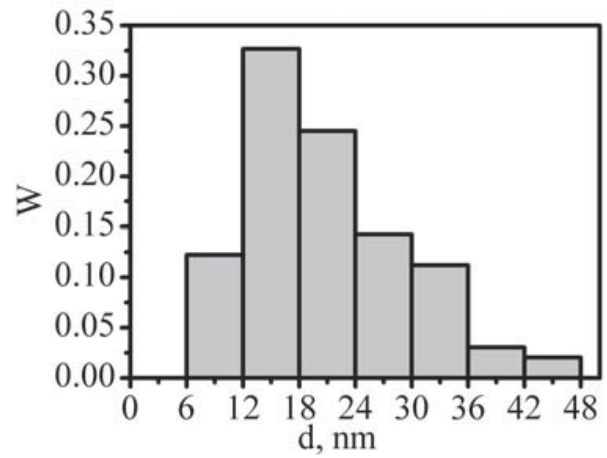

(f)

FIGURE 1. A concentration profile of the copper implanted by nitrogen ions (fluences: $\left.9 \times 10^{17} \mathrm{~cm}^{-2}(1), 18 \times 10^{17} \mathrm{~cm}^{-2}(2)\right)(a)$; the TEM image of the surface layer of copper implanted by nitrogen ions in the dark-field (b); and it's cross-section in the bright field (arrow 1 indicates the base material of the sample, arrow 2 - the ionic surface-modified layer, arrow 3-the nanopores) (c); microdiffraction patterns and the schemes of indexing (e); pores size distribution (d); grain size distribution (f) 


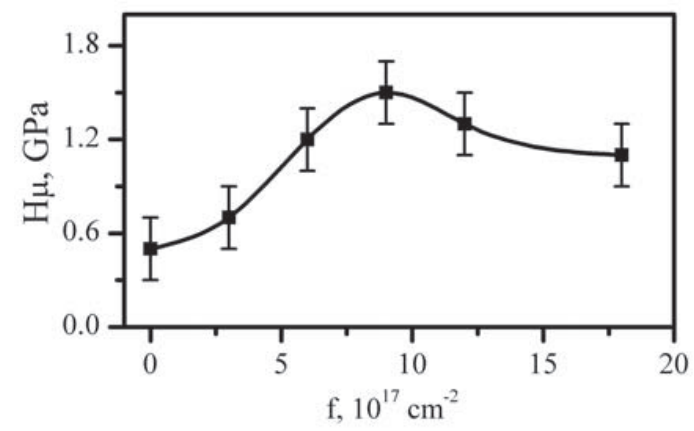

(a)

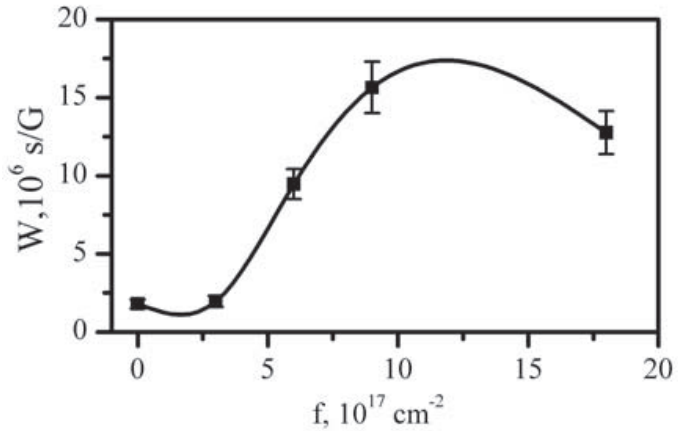

(b)

FIGURE 2. The microhardness $H$ (a), and the wear resistance $W$ (b) of the copper samples as a function of the nitrogen ion fluences $f$

The penetration depth of nitrogen ions in the copper samples was determined by secondary ions mass spectrometry of (VIMS) (MS-7201M, SELMI). The surface of the counterbody manufactured as a copper disk was not subjected to ion treatment. The wear resistance test was carried out in argon using the testing machine MIM-1 according to the "block-disk" scheme. The microhardness was measured using the nanohardness Nanotest-600.

\section{RESULTS AND DISCUSSION}

It was found by the VIMS method that the nitrogen concentration increases with fluences (Fig. 1(a)) up to 8 at.\% in the ion-modified surface layer (up to $120 \mathrm{~nm}$ in thickness).

The study of the structure of the copper samples after nitrogen ion bombardment by TEM showed that refinement (nanostructuring) of $\mathrm{Cu}$ and the formation of the $\mathrm{CuN}_{3}$ inclusions and the gas bubbles or pores in it occurred in the surface layer of the grain structure. These phenomena are observed at high fluences of ion irradiation in the case when its value was larger than $3 \times 10^{17} \mathrm{~cm}^{-2}$. When the fluences reached $9 \times 10^{17} \mathrm{~cm}^{-2}$, the grain cross size of the copper samples (3-30 $\mu \mathrm{m}$ in the initial state) decreased down to $0.01-0.1 \mu \mathrm{m}$ in the ion-implanted surface layer (Fig. 1(f)) and the cross dimension of the formed gas bubbles or pores was within the range from 2 to $40 \mathrm{~nm}$ (Fig. 1(e)).

It was established that a high-fluences implantation of nitrogen ions into the copper samples increases the wear resistance in 1.5-4.5 times depending on the duration of treatment during the work of a pair with the counterbody from copper in argon at the time of wear of $60 \mathrm{~min}$. We can see (Fig. 2) that the dependence of the wear resistance $W$ as a function of the ion irradiation fluences $f$ is close to directly proportional. The dependences of the microhardness on the surface layer of copper as a function of the fluencies of the nitrogen ions in $3 \times 10^{17} \mathrm{~cm}^{-2} \leq f \leq$ $9 \times 10^{17} \mathrm{~cm}^{-2}$ has the same character.

The improvement of the tribological properties of the friction pairs under high-nitrogen ion implantation may be associated with increased relaxation capacity of the surface layer as a result of a significant increase in the concentration of nitrogen in it, which is accompanied by the refinement of the grain structure (nanostructuring) of the main fcc-Cu phase and the formation of the $\mathrm{CuN}_{3}$ inclusions and nanopores or gas bubbles in the surface layer. In accordance with the Hall-Petch relationship [4], the decrease in the average grain size leads to the increase in the material microhardness. In addition to hardening, due to the nanostructuring of the surface layer, the $\mathrm{CuN}_{3}$ inclusions and nanopores can make a major contribution to increasing the wear resistance.

Owing to the small size, the nanopores can be inherently inclusions that retard the movement of dislocations. Indeed, the possibility of formation of spherical pores at the condensation of the vacancies along the dislocations was considered in [5]. The authors of this work have shown that such pores are very strong points of retardation of dislocations if their radius is equal or greater than 20 interatomic distances. They should cause hardening, if the distance between two pores is smaller than the free track length of a dislocation. In our case, the nanopores formed in the surface layer of the copper samples as a result of a high-fluences implantation of nitrogen are nothing more than an accumulation of vacancies with an average radius about 34 interatomic distances. 


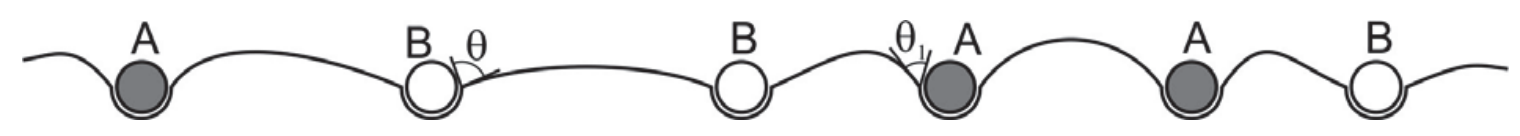

FIGURE 3. Scheme of retarding dislocations by $\mathrm{CuN}_{3}$ inclusions $(A)$ and nanopours $(B)$

Due to significant differences in the elasticity modulus of the material in nanopores, the moving dislocation rounds it (Fig. 3) [6], with the energy produced along the ledge boundary compensated by the elastic energy gain as the difference between the elastic modulus of both environments (matrix and nanopores) is large enough. There are large attraction forces near the $\mathrm{CuN}_{3}$ inclusions $A$ and nanopores $B$ (Fig. 3), so the dislocation line sharply deviates from them at points $A$ and $B$ where it forms the angle $\Theta$ almost equal to $\pi / 2$.

The curved dislocation line is less stable than the rectilinear one, and it always tends to become straight under the action of linear tension. Then the field of elastic tension is formed round the curved dislocation, which retards the movement of other dislocations moving in this direction. As a result, the interaction of dislocations with the $\mathrm{CuN}_{3}$ inclusions and nanopours leads to the formation of a strong barrier. To overcome this barrier, an additional energy is necessary, which causes strong hardening of the material.

Thus, it was shown by the TEM and VIMS methods that the improvement of the tribological properties of the implanted material can be associated with an increase in relaxation ability due to a significant increase in the nitrogen concentration in it, which is accompanied by refinement of the grain structure of the main phase of fcc-Cu and the formation of $\mathrm{CuN}_{3}$ inclusions and nanopores or gas bubbles in the ion-modified surface layer. A highfluence implantation of nitrogen ions in copper samples increases the wear resistance in 1.5-4.5 times together with a counterbody from the same material in the argon environment and the microhardness of the copper samples also increases.

\section{ACKNOWLEDGEMENTS}

The work was supported within the scope of the basic scientific research of state academies of sciences for 2013-2020 performed within the scope of state task "Science NRTPU" and at financial support of the Russian Fund of Basic Researches, the project No. 13-08-00616.

\section{REFERENCES}

1. V. J. Averbuh, K. A. Gamygin, Y. A. Gluskin, et al., Ferromagnetic Materials for Electric-Rotating Multichannel Contact Devices, Electrical Contacts (St. Petersburg State Polytechnic University, St. Petersburg, 2002).

2. V. E. Panin, V. P. Sergeev, and A. V. Panin, Nanostructuring of the Surface Layers of Materials of Construction and Application of Nanostructured Coatings (TPU Publ. House, Tomsk, 2008).

3. P. W. Leech, J. Mater. Sci. 22, 3139 (1987).

4. R. A. Andrievskii and A. M. Glazer, Phys. 179, 337 (2009).

5. P. Coulomb and J. Friedel, Dislocations and Mechanical Properties of Crystals: Lake Placid Conference, 1957, (Wiley, New York, 1957), pp. 555-576.

6. J. Friedel, Dislocations (Pergamon Press, Oxford, 1964). 